\title{
Radiation cross-linked gelatin/sodium alginate/ carboxymethylcellulose sodium hydrogel for the application as debridement glue paste
}

\author{
Yana Song ${ }^{1,2} \cdot$ Lin Xu $^{1} \cdot$ Ling X ${ }^{1,3} \cdot$ Li Deng $^{2}$
}

Received: 3 July 2020 / Revised: 8 November 2020 / Accepted: 19 December 2020 /

Published online: 7 January 2021

(ᄋ) The Author(s), under exclusive licence to Springer-Verlag GmbH, DE part of Springer Nature 2021

\begin{abstract}
Autolytic debridement can accelerate wound healing by removing necrotic tissue. A hydrogel was fabricated from an aqueous solution of gelatin, sodium alginate and carboxymethylcellulose sodium by radiation-induced cross-linking at room temperature, which was aiming at the application of debridement glue paste. The swelling ratio of the debridement glue paste is 30 times to its dry weight, when the weight ratio of gelatin/sodium alginate/carboxymethylcellulose sodium was 2:2:2 and the absorbed dose was $20 \mathrm{kGy}$, with dose rate of $20 \mathrm{~Gy} / \mathrm{min}$. The extrusion and compressive assay have confirmed that it possessed stable mechanical strength, and the weight ratio had little effect on the molecular structure by FTIR and TGA. Cell culture experiments demonstrated the debridement glue pastes with the cytotoxicity of grade 0 or 1 (biosafe). The debridement glue paste group could remove the necrotic tissue within 4 days and showed complete wound healing within 18 days; comparatively, the control group without treatment removed the necrotic tissue within 10 days and showed complete wound healing within 26 days in animal experiments using rabbit scald model. Histologic analysis exhibited that more granulation tissue was observed in debridement glue paste. The result of this study suggested that debridement glue pastes had excellent biocompatibility, could selectively remove necrotic tissue, induced granulation tissue formation and accelerated the wound healing.
\end{abstract}

Keywords Debridement glue paste $\cdot$ Swelling degree $\cdot$ Wound healing $\cdot$ Necrotic tissue $\cdot$ Radiation cross-linking

Ling Xu

lingxu@xmu.edu.cn

$\triangle$ Li Deng

dengli@mail.buct.edu.cn

Extended author information available on the last page of the article 


\section{Introduction}

Autolytic hydrogel can help to provide a moist wound environment and absorb wound exudate. The moist wound environment has shown benefit to little wounds by moistening necrotic eschars and facilitating their removal. Compared with the desiccated wound caused by air exposure, maintaining a moist wound environment has been shown to accelerate wound healing as much as 50\% [1]. A hard crust and a desiccation of the underlying collagen matrix and surrounding tissue at the wound edge was developed of dry wounds. Keratinocytes are forced to burrow below the surface of the crust and desiccated matrix as they only can migrate over viable tissues that supply nutrients necessary for their survival for occurring of the reepithelialization. This process takes considerable energy and time [2]. In contrast, keratinocytes can migrate easily and allow the formation of a matrix with a large amount of growth factors and other properties conducive to healing when the wound maintains a moist surface. Sodium alginate was biodegradable and dissolved in the exudate when it absorbed liquid swelling into gel; these functions had a significant effect on dry wound debridement, both to maintain a moist wound healing environment and to dissolve necrotic tissue and exudate together [3].

Polysaccharide polymers have the disadvantage of low stability and faster degradation in water. This could be avoided by introducing cross-linking in the system. To prepare debridement glue paste, several cross-linking methods have been developed. Radiation cross-linking is thought to be an ideal technique for preparing debridement glue paste without any cytotoxic additives. In this study, debridement glue paste was prepared by radiation cross-linking. Physical properties of the debridement glue paste such as the swelling behavior, mechanical strength and structural stability were investigated. Furthermore, the cytotoxicity was evaluated by the in vitro cell culture and the biocompatibility of the debridement glue paste was preliminary studied by the necrotic tissue removal assay and wound healing assay using New Zealand rabbits as the experimental animal.

\section{Experimental}

\section{Materials and animals}

Gelatin was obtained from $300 \mathrm{~g}$ Bloom, porcine, Type A, from Sigma Chemical Co. Ltd.; sodium alginate from pharmaceutical grade, from Qingdao Bright Moon Seaweed Group Co. Ltd., China; carboxymethylcellulose sodium from pharmaceutical grade, from Xi'an Yue Lai Medical technology Co. Ltd., China. Transparent polyurethane (TPU) dressing (Tegaderm, from 3 M Co. USA) was used in the wound healing and necrotic tissue removal assays as control. All the other reagents used here were all of analytical or equivalent grade. 
Specified pathogen-free male or female young adult New Zealand rabbits (weighing about $2.5 \mathrm{~kg}$ ) were used. The animals were fed specific standard laboratory food, maintained under sterile housing conditions and housed individually.

\section{Preparation of the debridement glue paste}

The debridement glue paste was typically prepared as follows: firstly, gelatin/sodium alginate/carboxymethylcellulose sodium powders with a different weight ratio of 2:1:3 (GAC2:1:3), 2:2:2 (GAC2:2:2) and 2:3:1 (GAC2:3:1) were dissolved in deionized water at $50{ }^{\circ} \mathrm{C}$ with a continuous stirring for $12 \mathrm{~min}$. The total polymer concentration was fixed to be $6 \%$. For homogenization, the mixture was further blended by an ARE-310 hybrid mixer (Japan Thinky Co., Ltd.) for $10 \mathrm{~min}$ and deaerated for $10 \mathrm{~min}$ to form a homogenous polymer slurry. Subsequently prepared solutions were filled into test tubes (inner diameter $10 \mathrm{~mm}$ ) or injector (the capacity of $5 \mathrm{ml}$ ) and subjected for $\gamma$-irradiation at a absorbed dose range of $20-40 \mathrm{kGy}$ using a ${ }^{60} \mathrm{Co}$ radiation facility, which was performed at room temperature at a dose rate range of 20-100 Gy/min ${ }^{4}$.

\section{Characterization of the debridement glue paste}

\section{Swelling behavior in deionized water and phosphate-buffered saline solution}

The debridement glue paste was squeezed from injector, the cylinders with diameter of $0.5 \mathrm{~mm}$ and length of $20 \mathrm{~mm}$, and then the samples were immersed in beakers containing $100 \mathrm{~mL}$ deionized water or phosphate-buffered saline (PBS) solution $\left(0.15 \mathrm{~mol} \mathrm{~L}{ }^{-1}, \mathrm{pH}=7.2\right)$ at $37{ }^{\circ} \mathrm{C}$. After soaking for desired time interval, the samples were withdrawn from the solution, gently removed surface solution by filter paper. The degree of swelling was calculated using Eq. (1). Three parallel samples were measured to achieve an average value.

$$
\text { Degree of Swelling }=\frac{m_{2}}{m_{1} \times 6 \%} \times 100 \%
$$

where $m_{1}, m_{2}$ represent weight of the sample before and after swelling and $6 \%$ represents the polymer concentration in the debridement glue pastes, respectively.

\section{Mechanical strength}

The compressive assay The compressive strength of samples was tested by the texture analyzer (TA.XT Plus, Stable Micro Systems, L td. UK). The cup (the capacity of $100 \mathrm{ml}$ ) was firmly fixed on the sample stage. And samples were not moved from the cup, and it was ensured that the height of the samples was identical by cutting at least $3 \mathrm{~cm}$ away from debridement glue paste surface. The flat plate probe $(\mathrm{P} / 0.52$ Delrin cylinder probe) with $5 \mathrm{~cm}$ of diameter was attached to the sample surface and moved downward vertically. The testing was performed with the pretest speed of 
$3.0 \mathrm{~mm} / \mathrm{s}$, the test speed of $1.0 \mathrm{~mm} / \mathrm{s}$, the post speed of $3.0 \mathrm{~mm} / \mathrm{s}$ and the drop height of $3 \mathrm{~cm}$, respectively. The test is repeated 6 times.

The extrusion assay The extrusion of samples was tested by a texture analyzer (TA. XT Plus, Stable Micro Systems, Ltd., UK). The injector (the capacity of $5 \mathrm{ml}$ ) with sample was fixed on a sample stage. The flat plate probe $(\mathrm{P} / 0.36$ Delrin cylinder probe) with the diameter of $3 \mathrm{~cm}$ was attached to the injector ram and to move vertically $[5,6]$. The testing conditions were consistent with that described above in 2.3.2.1. The test is repeated 6 times.

\section{Fourier Transform Infrared Spectroscopy (FTIR)}

The debridement glue paste was lyophilized and ground to fine powders. Fourier transform infrared spectroscopy (FTIR) analysis was performed using an FTIR spectrometer (Nicolet iNIO MX, Thermo Fisher Scientific, America). The scanning was performed with a resolution of $0.4 \mathrm{~cm}^{-1}$ and a range of $650-4000 \mathrm{~cm}^{-1}$. The number of scans was 16 .

\section{Thermogravimetric Analysis (TGA)}

Thermogravimetric analysis (TGA) was performed using the SDT Q600 (V20.9 Build 20) instrument, Nitrogen atmosphere flow rate $100 \mathrm{ml} / \mathrm{min}$, temperature $25-600{ }^{\circ} \mathrm{C}$, heating rate $20^{\circ} \mathrm{C} / \mathrm{min}$.

\section{In vitro cytotoxicity test (MTT assay)}

\section{Cell culture}

Briefly L929 fibroblasts cells were cultured in Dulbecco's modified Eagle's medium (DMEM) supplied with $10 \%$ FBS and $1 \%$ penicillin/streptomycin and incubated at $37{ }^{\circ} \mathrm{C}$ and $5 \% \mathrm{CO}_{2}$. The cells were detached by trypsin-EDTA when the cells attained $80 \%$ confluence.

\section{Cytotoxicity of the debridement glue paste extract $[7,8]$}

Based on the guideline of ISO 10993-5, the vitro cytotoxicity of the debridement glue paste was evaluated using the MTT assay. The extraction media were prepared in a standard culturing condition, and the sterilized debridement glue paste was incubated in a DMEM serum free medium with a weight/extraction medium ratio of $0.2 \mathrm{~g} / \mathrm{ml}$ for $24 \mathrm{~h}$. Subsequently, the mixture of DMEM and the debridement glue paste was filtered (0.22-ul sterile filter) and the fluid was withdrawn as the extraction medium. The extraction was diluted with DMEM medium to obtain extraction medium samples with concentration of $1,0.5$ and $0.1 \%$ (relative to the original extracts) before cytotoxicity testing. DMEM medium and 10\% DMSO dissolved in the DMEM medium were used as negative and positive controls, respectively [9]. 
L929 cells were incubated in a 96-well cell culture plate at a density of $1 \times 10^{4}$ cells per well for $24 \mathrm{~h}$. Then, the culture medium was removed and replaced with the prepared extraction medium and incubated for $72 \mathrm{~h}$. Then, $10 \mathrm{ul} \mathrm{MTT}(5 \mathrm{mg} / \mathrm{ml}$ in PBS) was pipetted into each well and incubated for $4 \mathrm{~h}$ at $37{ }^{\circ} \mathrm{C}$, then $100 \mathrm{ul} \mathrm{SDS}$ solution was added to each well followed by a 15-min oscillation. The absorbance was measured using a microplate reader (Bio-RAD680) at dual wavelength $(570 \mathrm{~nm}$ and $630 \mathrm{~nm}$ ). The relative growth rate (RGR) was defined in Eq. (2). Data were calculated from the average of six measurements and triplicate for the experiment [13].

$$
\mathrm{RGR}=\frac{\text { absorbance of sample }}{\text { absorbance of negative control }} \times 100 \%
$$

\section{Necrotic tissue removal and wound healing}

\section{Necrotic tissue removal}

Preoperatively, animals were administered anesthesia by ear vein injection of chloral hydrate $(3.5 \%, 30 \mathrm{mg} / \mathrm{kg})$. Under anesthesia, the dorsal hair was shaved and skin was disinfected thoroughly. To establish the scald model, two circular scalds $(\Phi 2.0 \mathrm{~cm})$ were created along the midline after marking the dorsal skin with a scald device (laboratory homemade) which possessed heated flat plate probe $(\Phi 2.0 \mathrm{~cm}$, thickness $0.5 \mathrm{~cm}$ ). The scald was created in temperature $80{ }^{\circ} \mathrm{C}$, time $60 \mathrm{~s}$. In gel group, the debridement glue paste was covered on the scald and then the wound beds were covered with TPU dressings, fixed with medical adhesive tape (MDS, China). In blank control group, wounds were treated with TPU dressing alone.

The debridement glue paste of wound was replaced every 2 days until necrotic tissue was removed absolutely; meanwhile, TPU dressing was replaced every 2 days for each animal in the control group.

\section{Wound healing}

As described in 2.5.1, wound healing of each animal was continued to be observed after removing necrotic tissues. In gel group, the wound beds were then filled with the optimized debridement glue paste chosen according to the previous test results and covered with TPU dressings. In blank control group, wounds were treated with TPU dressing alone. The debridement glue paste and TPU dressing of wounds were replaced every 2 days until wound was healing absolutely for each animal in the gel group and control group, respectively.

\section{Histological analysis}

The scald tissues of the necrotic tissue removal were harvested at $24 \mathrm{~h}$ after the operation. The surrounding tissues of debridement glue paste were harvested at 1 and 2 weeks after the operation, fixed in $4 \%$ paraformaldehyde in phosphatebuffered saline (PBS) solution for $48 \mathrm{~h}$, dehydrated and embedded in paraffin. 
Three-micrometer-thick sections were achieved with the Thermo Scientific HM315 Microtome. The sections were stained with hematoxylin-eosin (H\&E) and Masson trichrome and then observed under Olympus BX51 microscope. The images were captured and digitized for analyses with Image-Pro Plus software (Media Cybernetics, Ink, USA). The inflammatory response and neovascularization cell distribution of the debridement glue paste were reflected in the images taken at the magnifications of $4 \times[9]$.

\section{Results}

\section{Swelling behavior}

It is crucial to have sufficient swelling ability for debridement glue paste. Swelling behavior of the debridement glue pastes is shown in Fig. 1. In both deionized water and PBS, swelling isotherm of the debridement glue paste ascended rapidly initially and then maintained a maximum until $24 \mathrm{~h}$ (Fig. 1a and b). For the
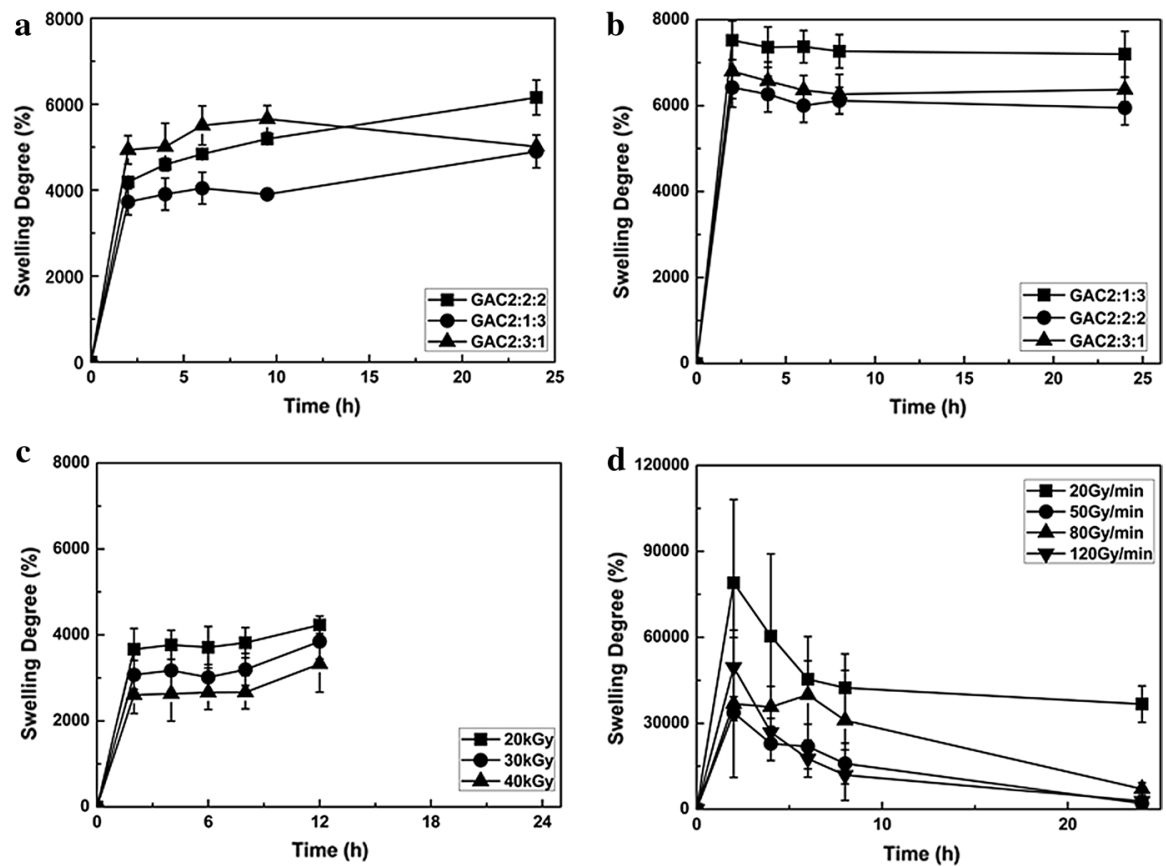

Fig. 1 Swelling degree of debridement glue paste in deionized water (a) and phosphate-buffered saline (PBS) solution (b), the weight ratios of G:A C =2:1:3 (GAC2:1:3), 2:2:2 (GAC2:2:2), 2:3:1 (GAC2:3:1). (c) Swelling degree of debridement glue pastes (GAC2:2:2) irradiated at different absorbed doses (20 kGy, $30 \mathrm{kGy}, 40 \mathrm{kGy}$ ) with the dose rate of $20 \mathrm{~Gy} / \mathrm{min}$ in deionized water. (d) Swelling degree of debridement glue pastes (GAC2:2:2) irradiated at $20 \mathrm{kGy}$ with the dose rate of $20 \mathrm{~Gy} / \mathrm{min}, 50 \mathrm{~Gy} / \mathrm{min}$, $80 \mathrm{~Gy} / \mathrm{min}$ and $120 \mathrm{~Gy} / \mathrm{min}$ in deionized water 
debridement glue pastes irradiated with different dose rates, the swelling degree increased rapidly within the initial $3 \mathrm{~h}$ and then decreased. All the debridement glue paste has the swelling ratio of 30 times as high as the pure debridement glue paste which is sufficient for the application as wound debridement glue paste [10].

The degree of swelling reached the equilibrium within $3 \mathrm{~h}$ in both deionized water and PBS. In the meantime, the equilibrium swelling degree increased as the alginate ratio increased (Fig. 1a) and the weight ratio of 2:2:2 (GAC2:2:2) could maintain the swelling degree a steady slow growth [11]. The swelling degree in PBS is higher than that in deionized water which manifested that the ions in PBS could not accelerate the hydrolysis of gelatin, sodium alginate and carboxymethylcellulose sodium. The weight ratios of 2:2:2 (GAC2:2:2) had a stable swelling degree indicating that the equivalent weight ratios of gelatin, sodium alginate and carboxymethylcellulose sodium have the beneficial on the swelling degree, so that the weight ratio of 2:2:2 was chosen for further experiment.

With the increase of the absorbed dose, the swelling degree of debridement glue paste decreased and tended to reach a constant value from 2 to $8 \mathrm{~h}$ when the absorbed dose was $20 \mathrm{kGy}, 30 \mathrm{kGy}$ and $40 \mathrm{kGy}$ (Fig. 1c). The cross-linking of the debridement glue paste also depends on the absorbed dose rate, the swelling degree of debridement glue paste prepared at the same weight ratios and dose decreased with the increasing of dose rate. The swelling degree increased rapidly initially and reduced when the dose rate was $20 \mathrm{~Gy} / \mathrm{min}, 50 \mathrm{~Gy} / \mathrm{min}, 80 \mathrm{~Gy} / \mathrm{min}$, and $120 \mathrm{~Gy} / \mathrm{min}$ (Fig. 1d). The main reason is that the composites finally disintegrated via hydrolysis, therefore an absorbed dose of $20 \mathrm{kGy}$ and dose rate of $20 \mathrm{~Gy} / \mathrm{min}$ was chosen for further experiment [12].
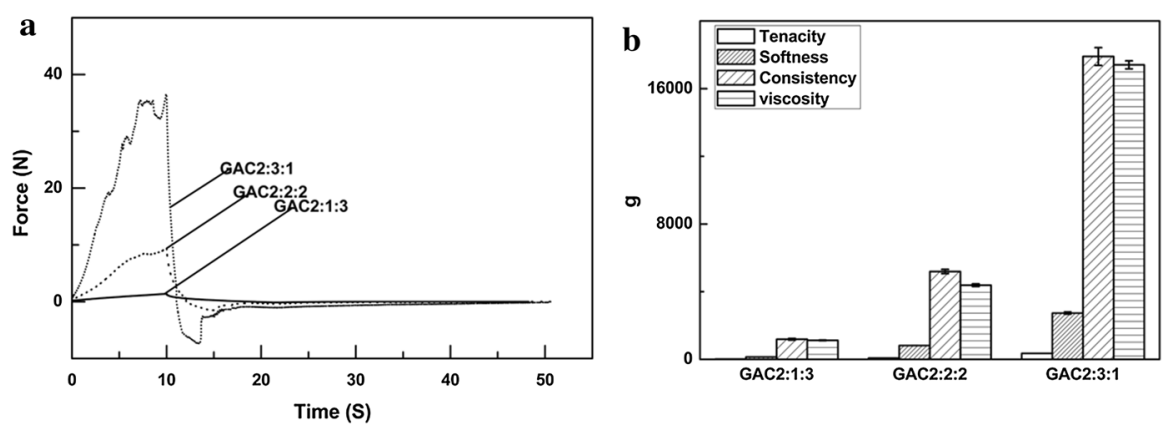

Fig. 2 a The compressive strength of debridement glue pastes with different weight ratios irradiated at $20 \mathrm{kGy}$; b the tenacity, softness, consistency and viscosity of debridement glue paste with different weight ratios irradiated at $20 \mathrm{kGy}$, the weight ratios of $\mathrm{G}: \mathrm{A}: \mathrm{C}=2: 1: 3$ (GAC2:1:3), 2:2:2 (GAC2:2:2), 2:3:1 (GAC2:3:1) 


\section{Mechanical strength}

\section{The compressive strength of debridement glue paste}

The debridement glue pastes were prepared to a stable mechanical form, which will benefit clinical and be easy to use. Debridement glue paste with different weights was prepared at $20 \mathrm{kGy}$, and the compressive strength was measured as shown in Fig. 2a. It could be seen that the compressive strength increases significantly with the increase in sodium alginate content. These results corresponded to the tenacity, softness, consistency and viscosity of debridement glue paste with different weight ratios, which is shown in Fig. $2 b$ that the tenacity, softness, consistency and viscosity of debridement glue paste increased when alginate was added into the system. In order to facilitate the clinical application of debridement glue paste, preparing the glue with a desirable compressive strength is necessary, and only the sample GAC2:2:2 which compressive strength was appropriate. The result is consistent with the result of swelling degree that the weight ratios of 2:2:2 were chosen for further experiment.

\section{The extrusion strength}

Because the shape of debridement glue paste was paste, the extrusion strength of debridement glue paste prepared at $20 \mathrm{kGy}$ was measured. As shown in Fig. 3a, the compressive strength increased significantly with the increase in the percentage of alginate. The extrusion curve fluctuated slightly in the start of the test; the main reason might be that there was a certain degree of friction between injector ram and injector wall, and the extrusion curve gradually became stable and reached a value when the testing speed was steady. The extrusion hardness of debridement glue paste irradiated at $20 \mathrm{kGy}$ with different dose rates was measured. As shown in Fig. 3b, the extrusion strength of glue, which was irradiated at $20 \mathrm{~Gy} / \mathrm{min}$, was the highest. This result demonstrated that higher dose rate would lead to a higher
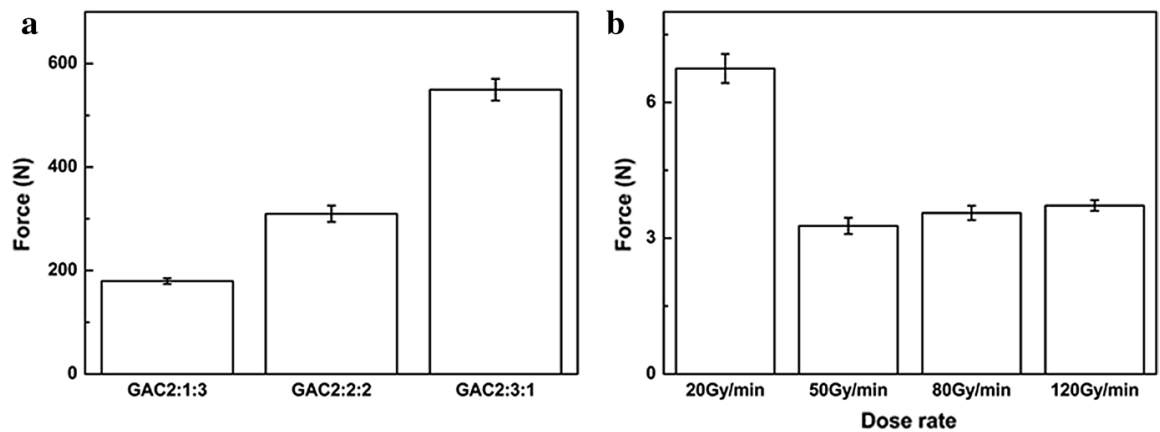

Fig. 3 The extrusion strength of debridement glue paste, with the weight ratios of G:A:C=2:1:3 (GAC2:1:3), 2:2:2 (GAC2:2:2), 2:3:1 (GAC2:3:1), irradiated at $20 \mathrm{kGy}$ with the dose rate of $20 \mathrm{~Gy} / \mathrm{min}$, is shown in (a). The hardness of debridement glue paste with different dose rates is shown in (b), the irradiated rate was $20 \mathrm{kGy}$ and the dose rate was $20 \mathrm{~Gy} / \mathrm{min}, 50 \mathrm{~Gy} / \mathrm{min}, 80 \mathrm{~Gy} / \mathrm{min}, 120 \mathrm{~Gy} / \mathrm{min}$ 


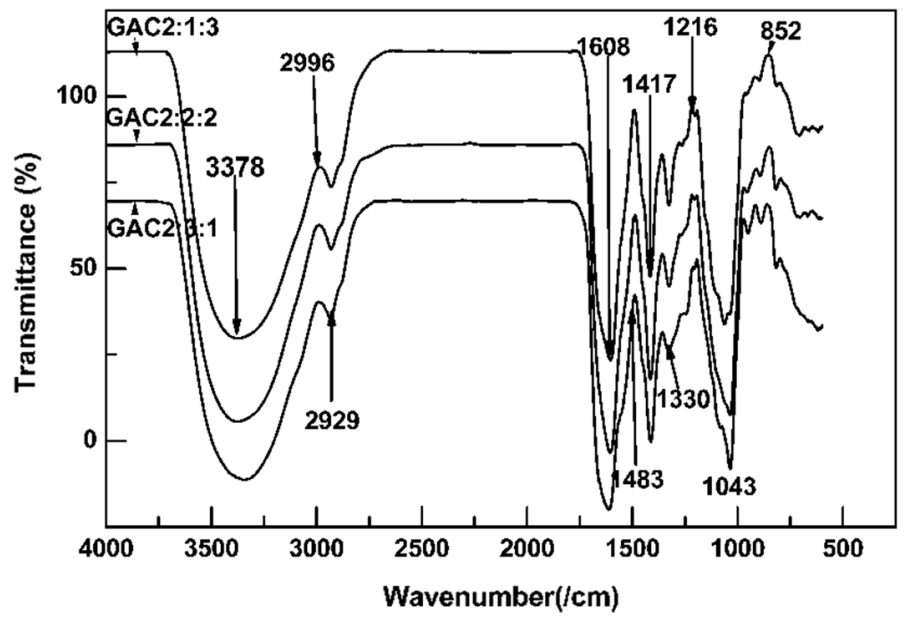

Fig. 4 FTIR spectra of debridement glue paste prepared at optimal conditions (absorbed dose $20 \mathrm{kGy}$, dose rate $20 \mathrm{~Gy} / \mathrm{min}$ ), the weight ratio of $\mathrm{G}: \mathrm{A}: \mathrm{C}=2: 1: 3$ (GAC2:1:3), 2:2:2 (GAC2:2:2), 2:3:1 $(\mathrm{GAC} 2: 3: 1)$

degree of degradation, resulting in the decrease in the extrusion strength of debridement glue paste. The reason for the relationship between extrusion strength and dose rate is not linear is deserved for further study. The varying trend of hardness of the debridement glue paste is kept consistent with the extrusion strength when dose rate increased at the same fixed dose (Fig. 4).

\section{Fourier Transform Infrared Spectroscopy (FTIR)}

The intermolecular interaction between components of the debridement glue paste was studied by FTIR spectra. The FTIR spectra of gelatin, sodium alginate and carboxymethylcellulose sodium composite debridement glue paste were obtained. The transmittance (\%) GAC2:1:3> GAC2:2:2> GAC2:3:1, the characteristic peaks, $852 \mathrm{~cm}^{-1}, 1216 \mathrm{~cm}^{-1}, 1483 \mathrm{~cm}^{-1}, 2929 \mathrm{~cm}^{-1}, 2996 \mathrm{~cm}^{-1}$ and $3378 \mathrm{~cm}^{-1}$, indicated that samples with different weight ratios could form the same intermolecular band during the cross-linking reaction. And the weight ratios of materials only have an effect on the strength of characteristic peak and have no influence on intermolecular band $^{[22]}$.

\section{Thermogravimetric analysis (TGA)}

As shown in Fig. 5, the debridement glue pastes, which were cross-linked by $\gamma$ irradiation, have a better thermal stability property than that without irradiation. The TGA curves showed that the degradation of debridement glue paste without irradiation started at a lower temperature compared with that of radiation cross-linked debridement glue paste. The thermogravimetric shows that the thermal stability of 


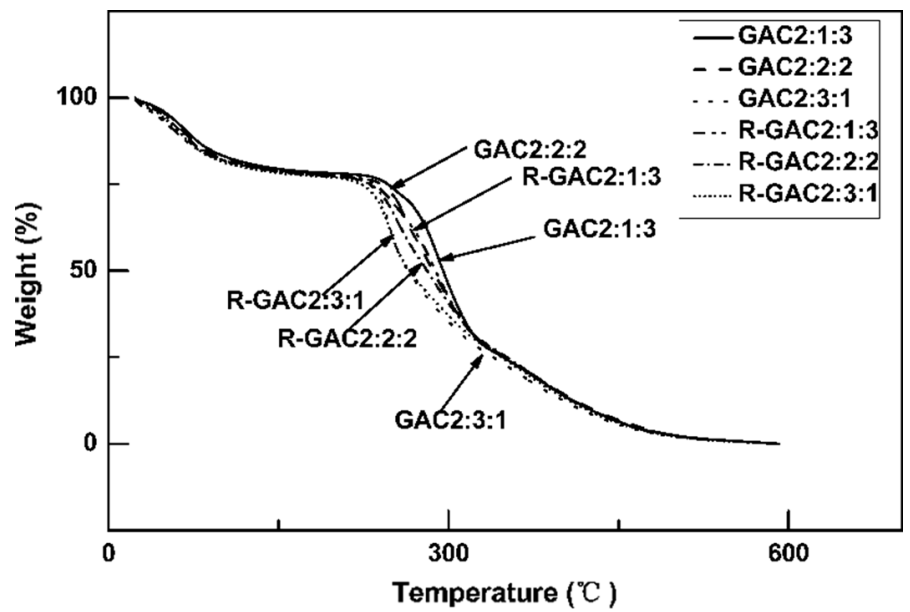

Fig. 5 Thermogravimetric curves for debridement glue pastes, the weight ratios of G:A:C=2:1:3 (GAC2:1:3), 2:2:2 (GAC2:2:2), 2:3:1 ( GAC2:3:1). R represents debridement glue paste prepared at optimal conditions (absorbed dose $20 \mathrm{kGy}$, dose rate $20 \mathrm{~Gy} / \mathrm{min}$ )

the debridement glue paste increased with the addition of sodium alginate as follows: GAC2:3:1 > GAC2:2:2> GAC2:1:3, R-GAC2:3:1 > R-GAC2:2:2 > R-GAC $2: 1: 3$. The result suggests the presence of cross-linking in the irradiation debridement glue paste. It can be assumed that the cross-linking between the anime group and carboxyl group of the debridement glue paste system would increase the thermal stability of the polymer complex. However, it was observed that GAC2:1:3 has the lowest thermal stability among the debridement glue paste without irradiation

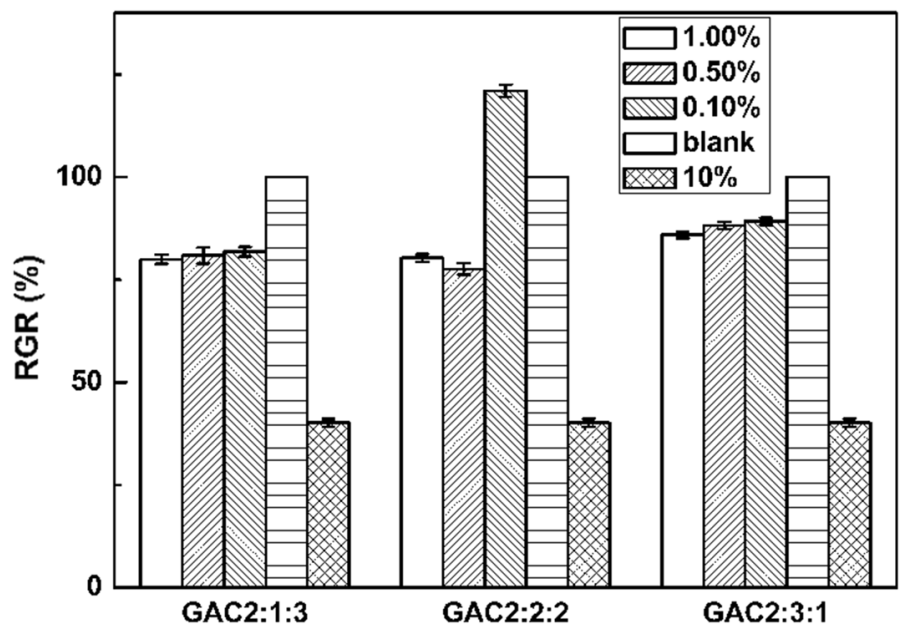

Fig. 6 Relative growth rate (RGR) of L929 in debridement glue pastes extraction medium after incubation. The debridement glue pastes were prepared at $20 \mathrm{kGy}$ and the weight ratios of G:A:C=2:1:3 (GAC2:1:3), 2:2:2 (GAC2:2:2), 2:3:1 (GAC2:3:1), respectively 
and R-GAC2:3:1 had the highest thermal stability among the irradiation debridement glue paste. Therefore, it can be concluded that there were other factors, as the weight ratios, of material which contributed to the thermal stability of debridement glue paste and the thermal stability could be a synthesized effect of all the factors (Fig. 6).

\section{In vitro cytotoxicity test (MTT assay)}

Cytotoxicity is an important property for biomaterials. Therefore, the cytotoxicity of the debridement glue paste was evaluated by MTT assay based on ISO 10993-5 standard. As shown in Fig. 7, after an incubation period of $72 \mathrm{~h}$, the RGRs of the entire sample were higher than $80 \%$ and could be classified to be scale 1 or 0 , which means that the material is safe to the L929 fibroblast cells. The result proved that the debridement glue paste composed of gelatin, sodium alginate and carboxymethylcellulose sodium possessed the excellent cyto-compatibility, which proved that the fabrication method was green and promising. The weight ratio of gelatin, sodium alginate and carboxymethylcellulose sodium had no significant influence on the RGRs suggesting that the materials were safe and could be used in further animal experiments studies.

\section{Necrotic tissue removal}

To investigate the main function in removing necrotic tissue and healing, the debridement glue paste prepared at optimal conditions was chosen for the necrotic tissue removal and healing experiment. The necrotic tissue was created by a heated flat plate probe $(\Phi 2.0 \mathrm{~cm}$, thickness $0.5 \mathrm{~cm})$ in $80^{\circ} \mathrm{C}$ and $60 \mathrm{~s}[13,14]$. Macroscopic observations of the necrotic tissue removal and wound healing treated by GAC2:2:2 debridement glue paste (a, c, e) at days 0, 4 and 18; control group (b, d, f) at days 0, 6 and 26 are shown in Fig. 7. Debridement glue pastes could avoid infection by using on the necrotic tissue in time. For the first few days after using the debridement glue paste, the necrotic tissue was dissolved gradually; the phenomenon can be explained that a moist environment was provided by debridement glue paste. The necrotic tissue of debridement glue paste group was removed, and the granulation was observed in day 4 after surgical operation; however, the necrotic tissue of control group still retained until day 10 after surgical operation, indicating that the debridement glue paste group showed a much higher necrotic tissue removing rate than control group. The debridement glue paste group could remove the necrotic tissue in 4 days and yet the control group finished the process after over 10 days. The following observation that in the debridement glue paste group wound (18 days) could heal in a shorter time than in control group (26 days), indicating that the presence of necrotic tissue would retard wound healing.

From the histological analyses of necrotic tissue as shown in Fig. 8a, b and Fig. 9a, b, little granulation, neovascularization and fibroblast were observed at 1 day after the surgical operation which proved that most of the living tissue had become necrotic tissue. At the 7 th day after the surgical procedure, the necrotic 
$\mathbf{a}$

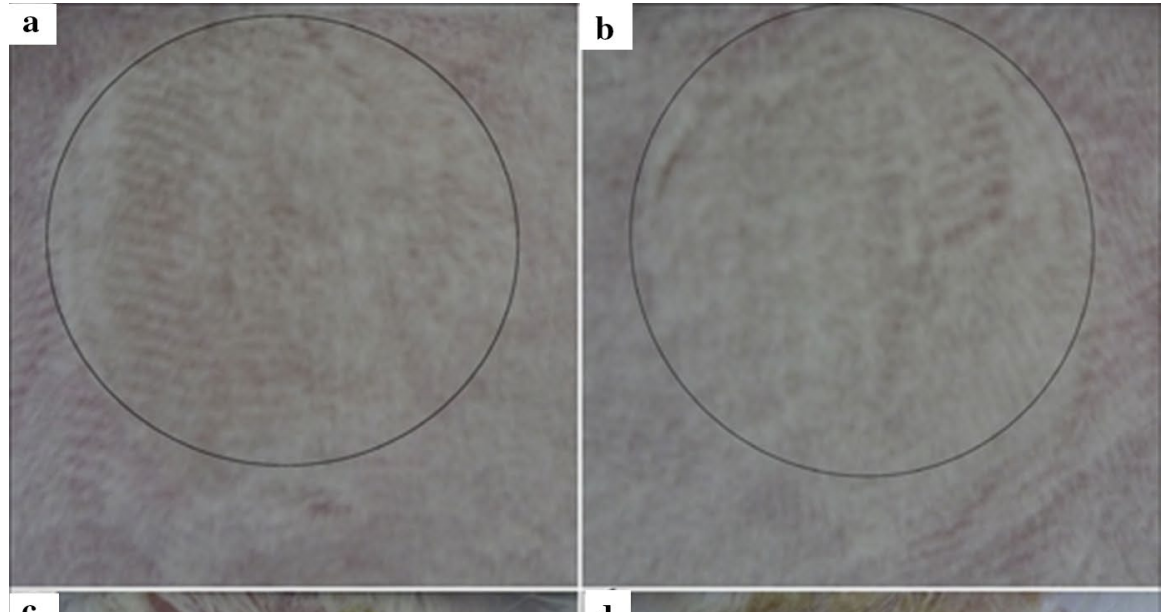

c

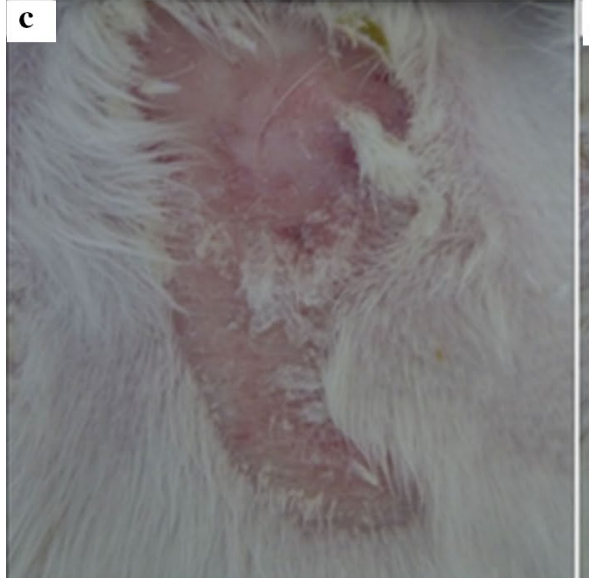

d

e

\section{f}

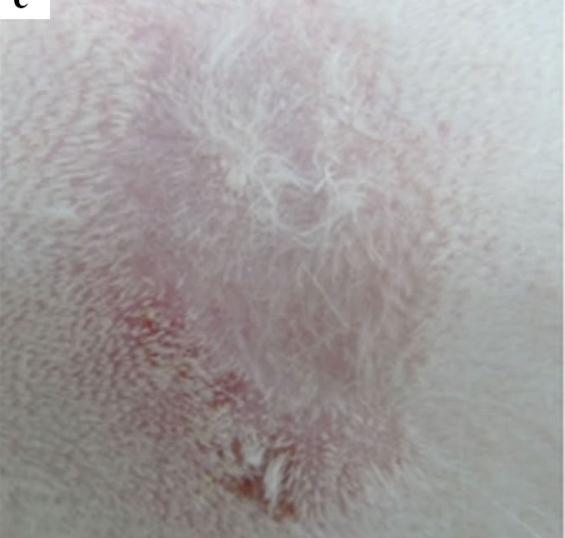

Fig. 7 Macroscopic observation of the necrotic tissue removal and wound healing treated by GAC2:2:2 debridement glue paste (a, c, e) at days 0, 4 and 18; control group $(\mathbf{b}, \mathbf{d}, \mathbf{f})$ at days 0,10 and 26 


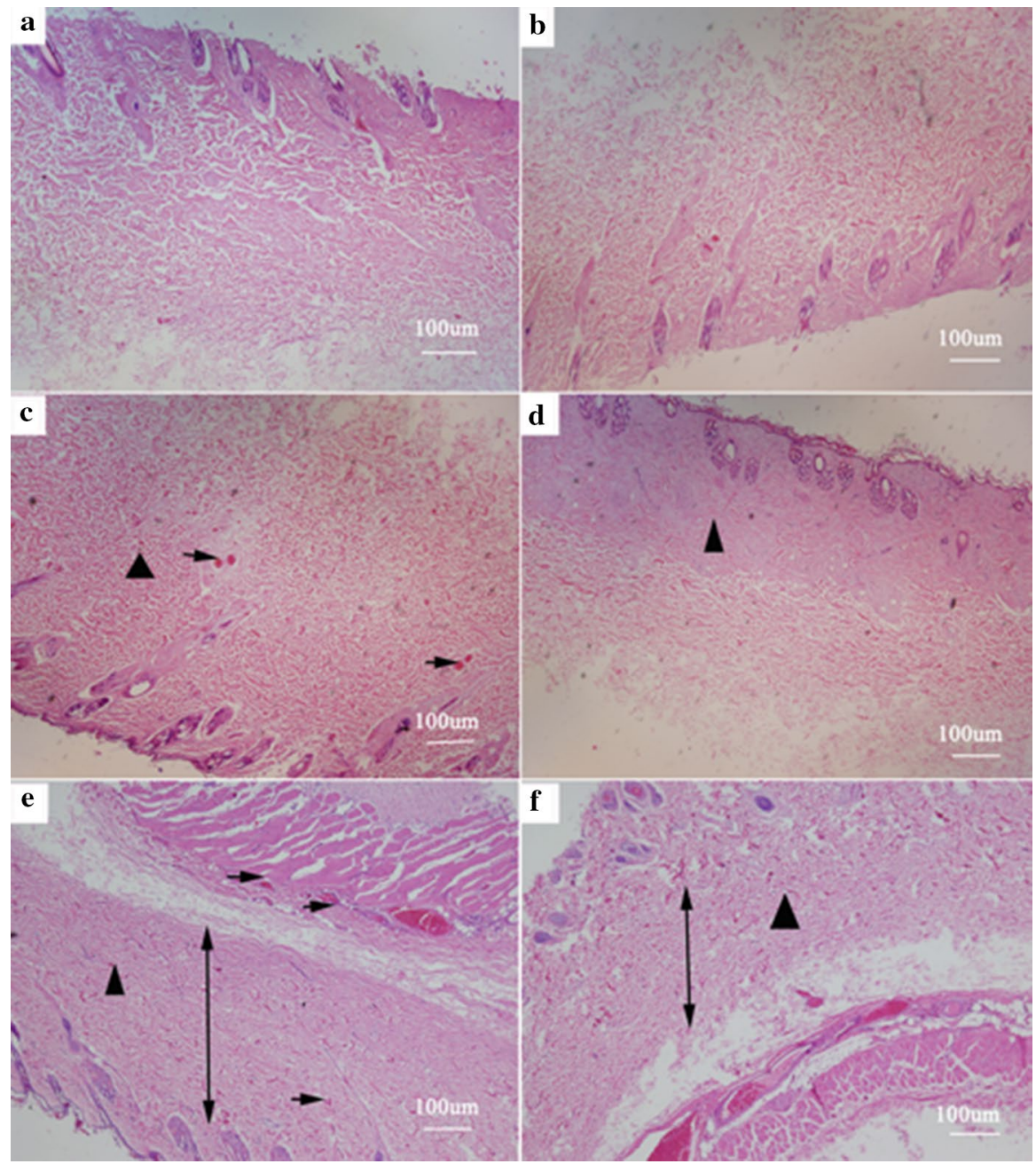

Fig. 8 Histologic analysis (H\&E) of the wound healing tissue, debridement glue paste (a, c, e) and control group (b, d, f) at days $1,7,14$. $\mathbf{\Delta}$ : peripheral granulation tissue; $\rightarrow$ : neovascularization. Double arrows show the distribution range of granulation tissue

tissue of debridement glue paste group was removed and the necrotic tissue of control group still remained. However, the status of the wounds was different although the wounds had not started to heal obviously; the regenerated granulation tissues filled up the wound in debridement glue paste group. In contrast, granulation tissue was not apparently regenerated in the blank control group. More granulation tissue was observed in debridement glue paste-treated group than in control group (Fig. 8c, $\mathrm{d}, \boldsymbol{\Delta}$ : peripheral granulation tissue). The same phenomenon was also observed at day 14 (Fig. 8e, f, $\boldsymbol{\Delta}$ : peripheral granulation tissue). Although the wound areas were similar in the groups, the histological analyses showed remarkable efficacy of 


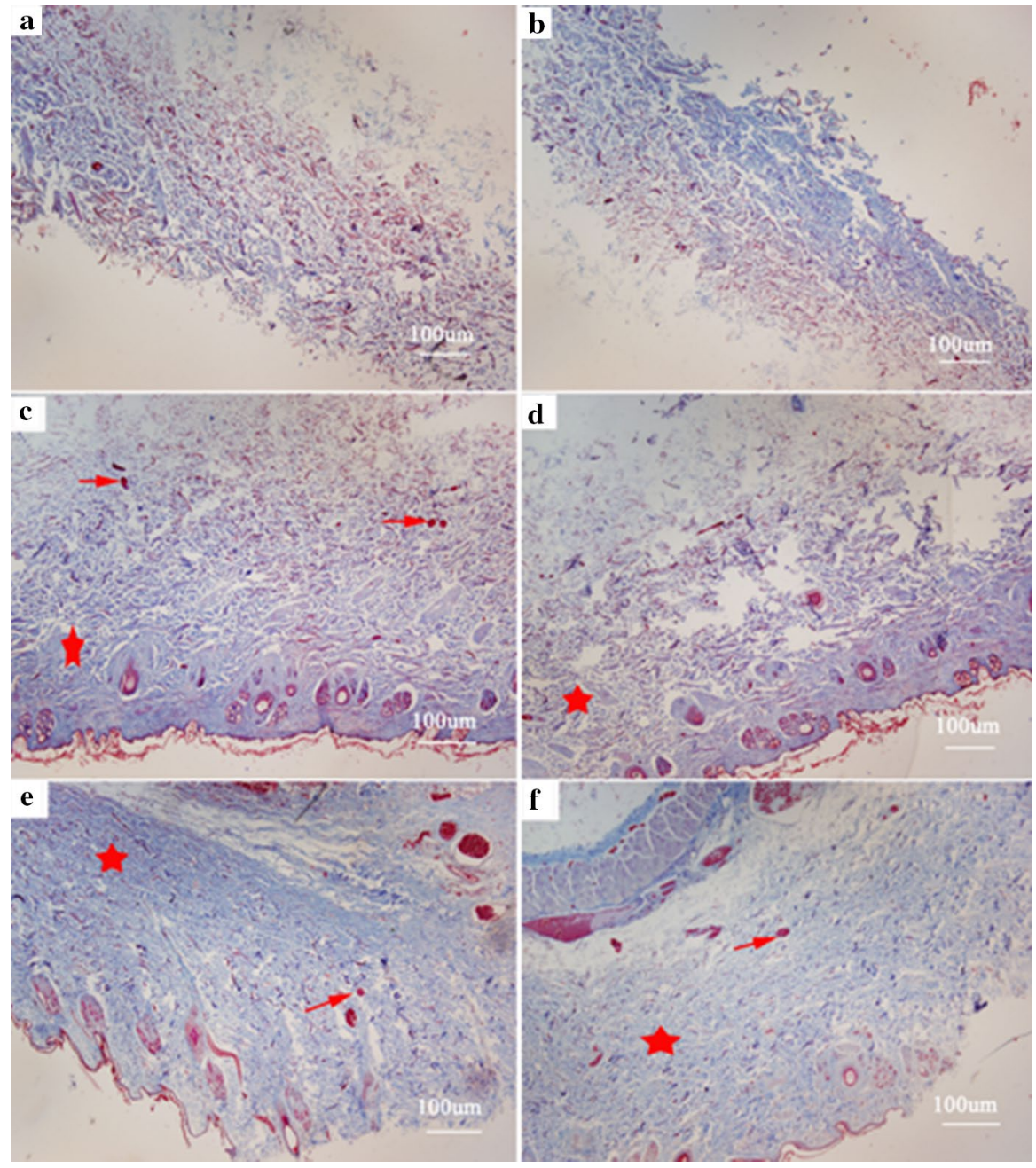

Fig. 9 Histologic analysis (Masson trichrome) of the wound healing tissue, debridement glue paste (a, c, e) and control group $(\mathbf{b}, \mathbf{d}, \mathbf{f})$ at days $1,7,14$. $\star$ : mature collagen fibers; $\rightarrow$ : neovascularization

debridement glue paste to enhance the granulation tissue formation. The distribution of granulation tissue was different. At day 14, debridement glue paste-treated wound showed an extensive distribution of the granulation tissue compared with blank control group (Fig. 8e, f, double arrows show the distribution range of granulation tissue). The application of debridement glue paste might provide a moist environment for transplantation and proliferation of fibroblasts which led to the formation of granulation tissue.

For the wound healing, collagen synthesis is a critical part. The collagen remodeling and maturation was highlighted in the Masson trichrome staining. In Masson trichrome staining, collagen type I (the major constituent in normal tissues) 
appeared to be thick and deep blue, whereas collagen type III appeared to be thin, light blue and reticular. At day 7 and 14, more thick, densely associated and organized collagen fibers were found in the debridement glue paste group, while thinner collagen fibers were appeared throughout the granulation tissue in the control group (Fig. 9 c-f; $\star$ : mature collagen fibers). At day 18, wound healing was completed and the epithelialization was smooth in the debridement glue paste, no keloids or hypertrophic scars were found in all animals. The complete wound closure was observed at day 26 in blank control group.

The signs of debridement glue paste keeping the wound a moist environment and promoting wound healing could be observed clearly; however, macroscopic signs of debridement glue paste reject the wound infection, drop, necrosis and serious inflammation were indiscernible throughout the experimental period. In the first several hours after the debridement glue paste was covered on the wound surface, the debridement glue paste began to swell as it absorbed the body fluid [13]. After four days, the debridement glue paste degraded gradually for reaching the maximum degree of swelling [15]. This behavior occurred in vivo when the body fluid was in the presence that was consistent with the swelling trend of the debridement glue paste in deionized water and phosphate-buffered saline solution [16, 17]. The degraded debridement glue paste need to instead in time to ensure the effective role of the debridement glue paste on wound healing.

Figure 7 depicts the macroscopic observation of the wounds treated with debridement glue paste and the control. The wound healing indicated that the debridement glue paste group showed a higher wound healing rate than the control group. The wound of the debridement glue paste group had finished healing at $18 \pm 2$ day and did not have the residues of scar, while the control group showed healing time of wound of $26 \pm 2$ day.

The debridement glue paste prepared by the method of radiation cross-linking could keep a moist wound healing environment and inhibit infection. In the meantime, it has the excellent mechanical strength and swelling degree. It could remove necrotic tissue effectively in necrotic tissue removal experiment and facilitate the wound healing, fibroblast proliferation and neovascularization so that the debridement glue paste group showed a higher wound healing rate than control group.

\section{Discussion and conclusion}

Various methods of wound debridement have been studied, but few focused on autolytic debridement which was the modality of the highest selectivity. Debridement glue paste in debridement can be frustrating and difficult for several reasons. First, most debridement biomaterials are not developed in debridement. Instead, they are mostly developed for use in simple wound healing studies. Furthermore, many methods of wound debridement have been existing, making autolytic debridement a challenge. In clinical, all kinds of wound in order to protect wound caused by the burn, trauma, operation, infection and other causes remove the foreign of body, promote the discharge of necrotic tissue, control wound infection, keep the wound be 
moist, have used different treatment means and product, but the ordinary methods and products are often unsatisfactory.

Debridement was the key step of wound healing that the suitable environment was benefit to wound healing. The traditional operation debridement was traumatic; a lot of undamaged tissues were nonselective sacrifice usually. The ordinary conservative debridement usually needed to a long time which was low efficiency. The enzymatic debridement also only can be selective debridement, less avail to the wound healing. Therefore, it needs to use other drugs or dressing for promoting wound healing which would increase the complexity of operation.

To be successful in using debridement, debridement glue paste needs to have mechanical properties that facilitate consistent use. The property depends on the preparation approach, for example, thermal heating, chemical cross-linking and radiation cross-linking. However, most of the cross-linking agents demonstrated toxicity in animal experiments. Although the treatment is effective in yielding which have a high degree of cross-linking by chemical reagents, the chemical residuals are highly cytotoxic which restrict their applications in the biomedical field. However, the radiation cross-linking is a green approach to fabricate debridement glue paste; the entire procedure of sample preparation is performed in a pure water system. Therefore, the prepared materials are additive-free. Several properties of debridement were tested that included swelling and compressive, several biocompatibility metrics and healing. Further, we also took into consideration the price of raw materials that may influence clinical application for debridement glue paste.

In terms of swelling degree, it is crucial to have sufficient swelling ability for debridement glue paste, because moist wound environment is beneficial to moisten necrotic eschars and facilitate their removal. The swelling property of debridement glue paste is an important factor. All the debridement glue paste has the swelling ratio of 30 times higher than the pure debridement glue paste which is sufficient for the application as wound debridement glue paste. Gelatin, sodium alginate and carboxymethylcellulose sodium all have a good solubility when immersed in aqueous solutions. For the anime group and carboxyl group interacted with each other via hydrogen band in the system, the swelling is obtained by the free $(-\mathrm{OH})$ group.

An additional requirement of debridement glue paste is that it is biocompatible. The debridement glue pastes were prepared to a stable mechanical form, which will benefit for clinical and be easy to be used. Finally, the requirement of debridement glue paste is biocompatible. First, the debridement glue pastes prepared by radiation cross-linking cannot be cytotoxic. Second, debridement glue pastes were implemented in eventual treatments must not elicit an immune response. Therefore, the cytotoxicity of the debridement glue paste was evaluated by MTT assay based on ISO 10993-5 standard. The RGRs suggested that the materials were safe and could be used in the further animal experiments studies.

To investigate the main function in removing necrotic tissue and healing, the debridement glue paste was chosen for the necrotic tissue removal and healing experiment. Wound healing is a complex process which consists of a series of events that happened at the moment of injury. The wound healing process mainly includes four stages: the hemostasis phase, the inflammatory, the proliferative phase and the remodeling phase $[18,19]$. The debridement glue paste prepared by 
the method of radiation cross-linking could keep a moist wound healing environment, inhibit infection, remove necrotic tissue effectively and facilitate the wound healing, fibroblast proliferation and neovascularization.

Gelatin, sodium alginate and carboxymethylcellulose sodium hybrid debridement glue pastes were prepared using a green fabrication method of radiation-induced cross-linking. The swelling behavior in deionized water and phosphate-bufferedsaline solution indicted that the debridement glue pastes had a higher swelling degree. The extrusion behavior from medical injector will facilitate for its clinical application. The optimal prepared conditions of debridement glue pastes were the weight ratios GAC2:2:2, absorbed dose $20 \mathrm{kGy}$ and dose rate $20 \mathrm{~Gy} / \mathrm{min}$. Cell culture experiments demonstrated that the debridement glue pastes had good cyto-compatibility with the cytotoxicity of grade 0 or 1 . The necrotic tissue removal experiment depicted that debridement glue pastes could remove necrotic tissue within a few days; meanwhile, it could accelerate the wound healing with thicker granulation and earlier reepithelialization. The comprehensive results of this study suggested their potential in the application of wound necrotic tissue removing materials.

Acknowledgments This research was financially supported by the Foundation for Xiamen high-level innovative talents plan, Fujian high-level innovative talents plan, Xiamen science and technology plan (Leading enterprise technological innovation project), Pilot Conversion Platform (No. 3502Z20121009), Amoy Industrial Biotechnology R\&D and Xiamen Gelfeel Biotechnology Co., Ltd. Following the guidelines of the Institutional Animal Care and Use Committee (IACUC), all animal experiments were performed at Peking University under authorization No. CDE-XuL-001.

\section{References}

1. Geronemus RG, Robins P (1982) The effect of two new dressings on epidermal wound healing[J]. J dermatol surg 8(10):850-852

2. Woodley DT, Chen JD, Kim JP et al (1993) Re-epithelialization: Human keratinocyte locomotion[J]. Dermatol clin 11(4):641-646

3. Nemeth AJ, Eaglstein WH (1993) Wound dressings and local treatment in leg ulcers: Diagnosis and treatment $[\mathrm{J}]$. Leg Ulcers: Diagnosis and Treatment. Elsevier, Amsterdam, pp 325-333

4. Zhou Y, Xu L, Zhang X et al (2012) Radiation synthesis of gelatin/CM-chitosan/ $\beta$-tricalcium phosphate composite scaffold for bone tissue engineering[J]. Mater Sci Eng, C 32(4):994-1000

5. Segura T, Anderson BC, Chung PH et al (2005) Crosslinked hyaluronic acid hydrogels: a strategy to functionalize and pattern[J]. Biomaterials 26(4):359-371

6. He L, Xueming X, Shidong G (2007) Rheological and textural properties of full-fat and low-fat cheese analogues[J]. Trans Chin Soc Agric Eng 23(5):32-41

7. Kasahara H, Tanaka E, Fukuyama N et al (2003) Biodegradable gelatin hydrogel potentiates the angiogenic effect of fibroblast growth factor 4 plasmid in rabbit hindlimb ischemia[J]. J Am Coll Cardiol 41(6):1056-1062

8. Nair S, Remya NS, Remya S et al (2011) A biodegradable in situ injectable hydrogel based on chitosan and oxidized hyaluronic acid for tissue engineering applications[J]. Carbohyd Polym 85(4):838-844

9. Huang X, Zhang Y, Zhang X et al (2013) Influence of radiation crosslinked carboxymethyl-chitosan/gelatin hydrogel on cutaneous wound healing[J]. Mater Sci Eng, C 33(8):4816-4824

10. Dawes-Higgs EK, Swain MV, Higgs RJED et al (2004) Accuracy and reliability of a dynamic biomechanical skin measurement probe for the analysis of stiffness and viscoelasticity[J]. Physiol Meas 25(1):97 
11. Hiroki A, Tran HT, Nagasawa N et al (2009) Metal adsorption of carboxymethyl cellulose/carboxymethyl chitosan blend hydrogels prepared by Gamma irradiation[J]. Radiat Phys Chem 78(12): 1076-1080

12. Kim S, Nimni ME, Yang Z et al (2005) Chitosan/gelatin-based films crosslinked by proanthocyanidin[J]. J Biomed Mater Res B Appl Biomater 75(2):442-450

13. Maas-Irslinger R, Hensby CN, Farley KL (2003) Experimental methods to demonstrate the efficacy and safety of Xenaderm (TM) ointment: A novel formulation for treatment of injured skin due to pressure ulcers[J]. Wounds-a compendium of clinical research and practice 15(3):2S-8S

14. König M, Vanscheidt W, Augustin M et al (2005) Enzymatic versus autolytic debridement of chronic leg ulcers: a prospective randomised trial[J]. J wound care 14(7):320-323

15. Wang T, Zhu XK, Xue XT et al (2012) Hydrogel sheets of chitosan, honey and gelatin as burn wound dressings[J]. Carbohyd Polym 88(1):75-83

16. Metcalfe AD, Ferguson MWJ (2007) Bioengineering skin using mechanisms of regeneration and repair[J]. Biomaterials 28(34):5100-5113

17. Brown RA, Phillips JB (2007) Cell responses to biomimetic protein scaffolds used in tissue repair and engineering $[\mathrm{J}]$. Int Rev Cytol 262:75-150

18. Kondo T, Ishida Y (2010) Molecular pathology of wound healing[J]. Forensic Sci Int 203(1):93-98

19. Enoch S, Leaper DJ (2008) Basic science of wound healing[J]. Surgery (Oxford) 26(2):31-37

Publisher's Note Springer Nature remains neutral with regard to jurisdictional claims in published maps and institutional affiliations.

\section{Affiliations}

\section{Yana Song ${ }^{1,2} \cdot$ Lin $\mathrm{Xu}^{1} \cdot$ Ling $\mathrm{Xu}^{1,3} \cdot$ Li Deng $^{2}$}

1 State Key Laboratory of Molecular Vaccinology and Molecular Diagnostics, School of Public Health, and National Institute of Diagnostics and Vaccine Development in Infectious Diseases, Xiamen University, Xiamen 361005, People's Republic of China

2 Beijing Bioprocess Key Laboratory, College of Life Science and Technology, Beijing University of Chemical Technology, Beijing 100029, People's Republic of China

3 Xiamen Gelfeel Biotechnology Co., Ltd, Xiamen 361022, People's Republic of China 